\title{
Modeling the Failure Behavior of CFRP Laminates Subjected to Combined Thermal and Mechanical Loadings
}

\author{
Weina Zhao*, Hongwei Song ${ }^{\dagger, \S}$ and Chenguang Huang ${ }^{\ddagger}, \S$ \\ Key Laboratory for Mechanics in Fluid Solid Coupling System \\ Institute of Mechanics, Chinese Academy of Sciences \\ Beijing 100190, P. R. China \\ School of Engineering Science \\ University of Chinese Academy of Sciences \\ Beijing 100049, P. R. China \\ *zhaoweina@imech.ac.cn \\ †songhw@imech.ac.cn \\ †huangcg@imech.ac.cn \\ Yihui Huang \\ Ningbo Institute of Materials Technology and Engineering \\ Chinese Academy of Sciences, Ningbo 315201, P. R. China \\ huangyihui@nimte.ac.cn
}

Received 9 May 2016

Revised 17 February 2017

Accepted 22 March 2017

Published 17 May 2017

This paper proposes a theoretical approach to predict the failure behavior of laminated carbon fiber reinforced polymer (CFRP) under combined thermal and mechanical loadings. Two types of CFRP Laminates, i.e., CCF300/BA9916 and T700/BA9916, are investigated, and TGA tests in both nitrogen and oxidation environments at different heating rates are carried out to obtain the thermal decomposition kinetic parameters of polymer matrix and carbon fiber. Based on the thermal decomposition behavior and a multi-level structure model, the thermal physical properties, mechanical properties and thermal deformations of the laminated composites at high temperatures are obtained. Then substituting thermally degraded properties into constitutive equations of composite materials as macroscopic defects, the damage mode and failure strength of the laminated composite under thermo-mechanical loadings is obtained. Predicted elastic properties and failure strength are compared with experimental results as well as previous models. Effects of heating rates and heating environments through rigorous physical model are considered in the present work. It is found that the heating rate significantly affects the thermal and mechanical properties, the higher the heating rate, the less degraded are the thermo-mechanical properties and failure strength at a given temperature. Young's modulus and failure strength of T700/BA9916 are higher than those of CCF300/BA9916

$\S^{\S}$ Corresponding authors. 


\begin{abstract}
at high temperatures, due to the higher volume fraction of carbon fibers, which are less
\end{abstract} weakened in thermal environment.

Keywords: Carbon fiber reinforced polymer; laminated composite; thermo-mechanical loadings; thermal decomposition; pyrolysis; failure.

\title{
1. Introduction
}

Due to superior characteristics such as light weight, high specific strength, high specific stiffness, improved chemical resistance and temperature tolerance, carbon fiber reinforced polymer (CFRP) is being increasingly used in industrial sectors, e.g., automotive, pressure vessels, civil engineering as well as aerospace and military. During service, CFRP composites are usually or accidentally subjected to the combination of thermal and mechanical loadings. When a laminated CFRP composite based on polymer matrix is exposed to elevated or high temperatures, complex chemical process and physical process may take place. The chemical process involves viscous softening, melting, decomposition and gasification of the polymer matrix and carbon fibers; formation, growth and oxidation of char; oxidation of carbon fibers. The physical process involves thermal expansion and contraction, thermally-induced strains, fiber-matrix interfacial debonding, matrix cracking and delamination damage [Mouritz et al., 2009; Dimitrienko, 1999]. As a result, the mechanical behavior of CFRP composites at high temperatures demonstrates a number of peculiarities such as intensive degradation of mechanical properties, dependence of mechanical characteristics, e.g., elastic modulus, strength, not only on temperature but also on the time exposed to high temperatures, or heating rates, etc. [Dimitrienko, 1999; Bahramian et al., 2006; Feih et al., 2007]. Therefore, the failure behavior of CFRP laminates under thermo-mechanical loading is very complicated and illusive to model.

Most previous studies focus on the fire behavior of composites and thermomechanical effects on composites. As Mouritz et al. [2009] commented, until recently the major progress in the modelling and measurement of fire reaction properties of composite materials has not been matched by advances in the analysis of their structural behavior in fire. Kandare et al. [2012] developed a model to predict the temperature, decomposition, softening and failure of laminates with an intumescent coating in fire. Feih et al. [2007] predicted the time-to-failure of laminates loaded in tension or compression and exposed to one-sided radiant heating by fire. Bai et al. [2008] proposed a one-dimensional model to predict the thermal responses of fiber reinforced polymer (FRP) composites in time and space domain up to high temperatures, based on temperature-dependent thermo-physical property models developed previously. Later on, a thermomechanical model considering temperaturedependent mechanical property of the Young's modulus, viscosity and coefficient of thermal expansion is presented for predicting the time-dependent deflections of cellular FRP slab elements subjected to mechanical loading and fire from one side [Bai and Keller, 2009]. Zenkour et al. [2013] examined the bending response of an 
orthotropic rectangular plate depending on two-parameter elastic foundations under thermo-mechanical loadings.

Although the degradation behavior of mechanical properties of FRP composites in high-temperature is a complex phenomenon, it has been found that the glass transition temperature of resin, $T g$ and decomposition temperature of resin, $T d$ are important indexes in modeling the degraded mechanical properties [Bai et al., 2008; Kandare et al., 2012; Gibson et al., 2006]. Several models have been developed to predict the degraded mechanical behavior of FRP materials made from different fibers and with different manufacturing procedures at elevated and high temperatures. Most of these models comprise the index temperature $\mathrm{Tg}$ and involve empirical or statistical curve fitting procedures. Mahieux and Reifsnider [Mahieux and Reifsnider, 2001] developed a theoretical model for temperature-dependent properties, and Weibull functions were used to describe the change in modulus over the full temperature range including $T g$. The model can successfully represent the behavior of following composites: PMMA, PEEK, PPS and AS4/PPS composite. Bisby [Bisby et al., 2005] proposed a sigmoid function which describes the reductions in strength, elastic modulus, and bond strength for FRP composites for temperatures up to about $800^{\circ} \mathrm{C}$. Gibson et al. [2006] put forward a general model that takes the form of a hyperbolic tangent function, and considers the extent of resin decomposition. The model is derived from the glass/polyester laminate exposed to one-sided heat flux for elevated temperature up to $200^{\circ} \mathrm{C}$. Wang et al. [2011] proposed a segmented model to describe the tensile strength of CFRP pultruded strips at high temperatures. The parameters of the model under different temperature ranges are based on the test data. Rigorous theoretical models that contain accurate physicalchemical processes, specifically, reveal the significant difference of various heating rate and heating environment, are still insufficient.

Unlike most previous studies, which examine the cases in fire conditions and typically subjected to one-side heating, the present work examines the case that laminated CFRP composites are uniformly heated while subjected to mechanical loading. The study is beneficial to understand the degradation behavior in mechanical properties and failure behavior of CFRP in high temperature environments. It has been widely recognized that at room temperature laminated composites failed in a progressive way when subjected to the mechanical loading. Huang [2004] describes a micromechanical prediction procedure to simulate the progressive failure strength of a composite laminate subjected to multi-axial loading. A bridging model is utilized to define the instantaneous stiffness matrix and to relate the stress increments in the constituent fiber and resin materials of the lamina with those stress increments exerted on it. Based on the model, the corresponding failure mode can be identified automatically. Soden et al. [1998] give the detailed consideration of different selected types of composites, and predict effects of lay-up, layer thicknesses, stacking sequences, and loading conditions to the strength of composite laminates. Gotsis et al. [1998] used two computer codes to predict the first-ply failure and 
fracture in composite laminates under biaxial loading. Li et al. [2002] developed an improved technique for predicting the failure of composite materials considering residual stress. Recently, Gadade et al. [2016a] analyzed the ultimate failure analysis of laminated composites subjected to biaxial loadings. They obtained the first-ply failure (FPF) and last-ply failure (LPF) stress envelops by using Puck's failure criterion. Compared with classical Tsai-Wu, Tsai-Hill and Lee's failure criteria, it can be concluded that the classical criterions are more conservative than Puck's failure criterion. They also used Puck's failure criteria to investigate the second order statistics of initial and final failure of laminated composites subjected to hygrothermo-mechanical loadings [Gadade et al., 2016b]. Results showed that predicted thermo-mechanical strengths of laminates are in good agreement with those experimental data. It is an interesting subject whether the degraded high-temperature CFRP laminate still fail in a progressive damage way under the combined thermal and mechanical loading.

A systematic approach is developed to model the failure behavior of laminated CFRP composites subjected to combined thermal and mechanical loadings in the present work. A mutli-level structure model including thermal decomposition of both polymer matrix and carbon fiber at high temperature is incorporated into the progressive damage analysis of laminated CFRP composites under mechanical loading. Thermal gravimetric analysis (TGA) tests of composites in both nitrogen and oxidation environments at different heating rates are carried out to find the thermal decomposition kinetic parameters of the polymer matrix and the carbon fiber. Based on the thermal decomposition behavior, the thermal physical properties, mechanical properties and thermal deformations of the laminated CFRP at high temperatures are obtained. The decomposed material properties are taken as defects in the stiffness matrix, and the stress state of each single layer is calculated when the laminated CFRP subject to tensile or compressive load. Progressive damage modes and failure loadings of the composite are subsequently obtained, and failure behaviors are compared with experimental results.

\section{Outline of General Approaches}

\subsection{Experimental observation}

In the present study, two types of CFRP laminates, i.e., CCF300/BA9916 and T700/BA9916, subjected to thermo-mechanical loadings are investigated. Laminated composites are in the symmetric ply pattern of $\left[45^{\circ} / 0^{\circ} /-45^{\circ} / 90^{\circ}\right]_{2 \mathrm{~S}}$, and the thickness of each lamina is $0.125 \mathrm{~mm}$ for CCF300/BA9916 and $0.150 \mathrm{~mm}$ for T700/BA9916, making the total thickness of CFRP laminate $2.00 \mathrm{~mm}$ and $2.40 \mathrm{~mm}$, respectively. The fiber volume fraction is $\varphi_{f}^{0}=0.420$ for CCF300/BA9916 and $\varphi_{f}^{0}=0.533$ for T700/BA9916.

When such a CFRP laminate is subjected to thermo-mechanical loadings, the failure behavior is different from that at room temperature: composites undergo 


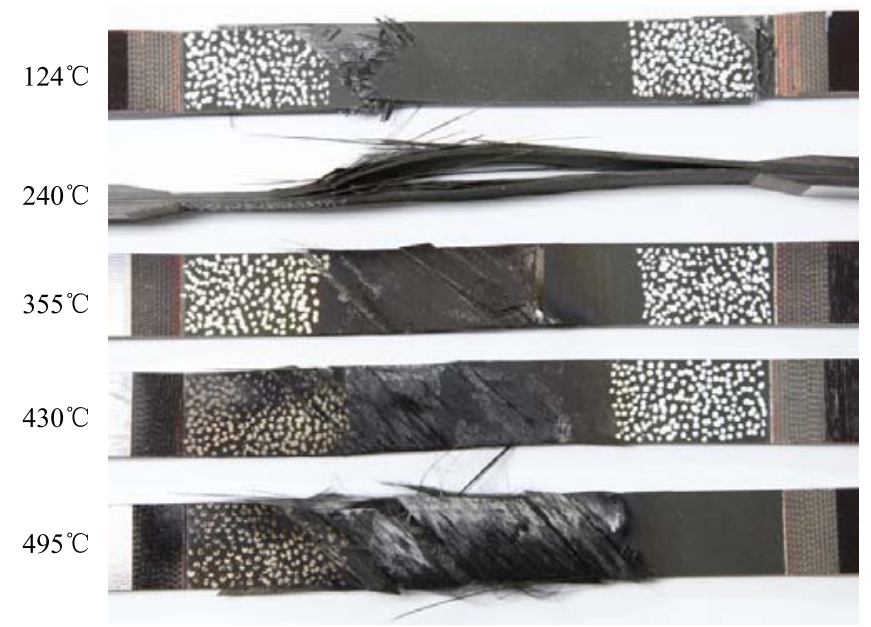

(a)

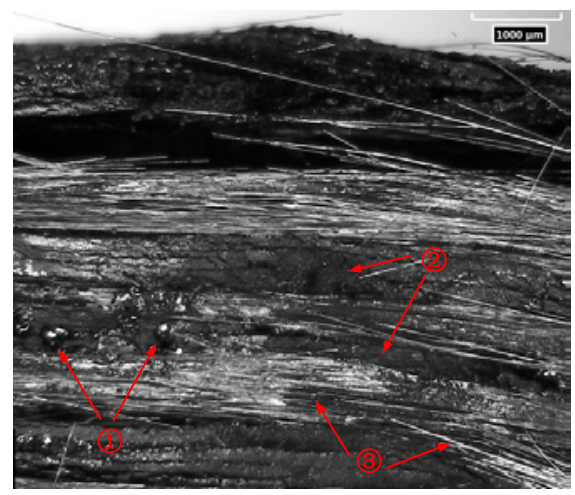

(b)

Fig. 1. Tensile failure modes of T700/BA9916 at elevated and high temperatures (a) failed specimens; (b) morphology of temperature $430^{\circ} \mathrm{C}$ : (1) melted matrix, (2) residue char, (3) broken fibers.

thermochemical decomposition and irreversible changes of thermos-mechanical properties, which adversely affect the structural integrity and reliability of the composite, as well as the elastic property and failure strength. Figure 1 illustrates the tensile failure mode of T700/BA9916 at elevated and high temperatures. As temperature rises, polymer matrix is softened and melted, and the bonding strength due to matrix is weakened. With the acceleration of matrix pyrolysis and fiber oxidation at high temperature, intra-laminar and inter-laminar cracks are formed and propagated, which result in significant degradation in mechanical properties. In Fig. 1(b), melted matrix, broken bare fibers and residue char can be spotted for the specimen failed at the temperature of $430^{\circ} \mathrm{C}$. 


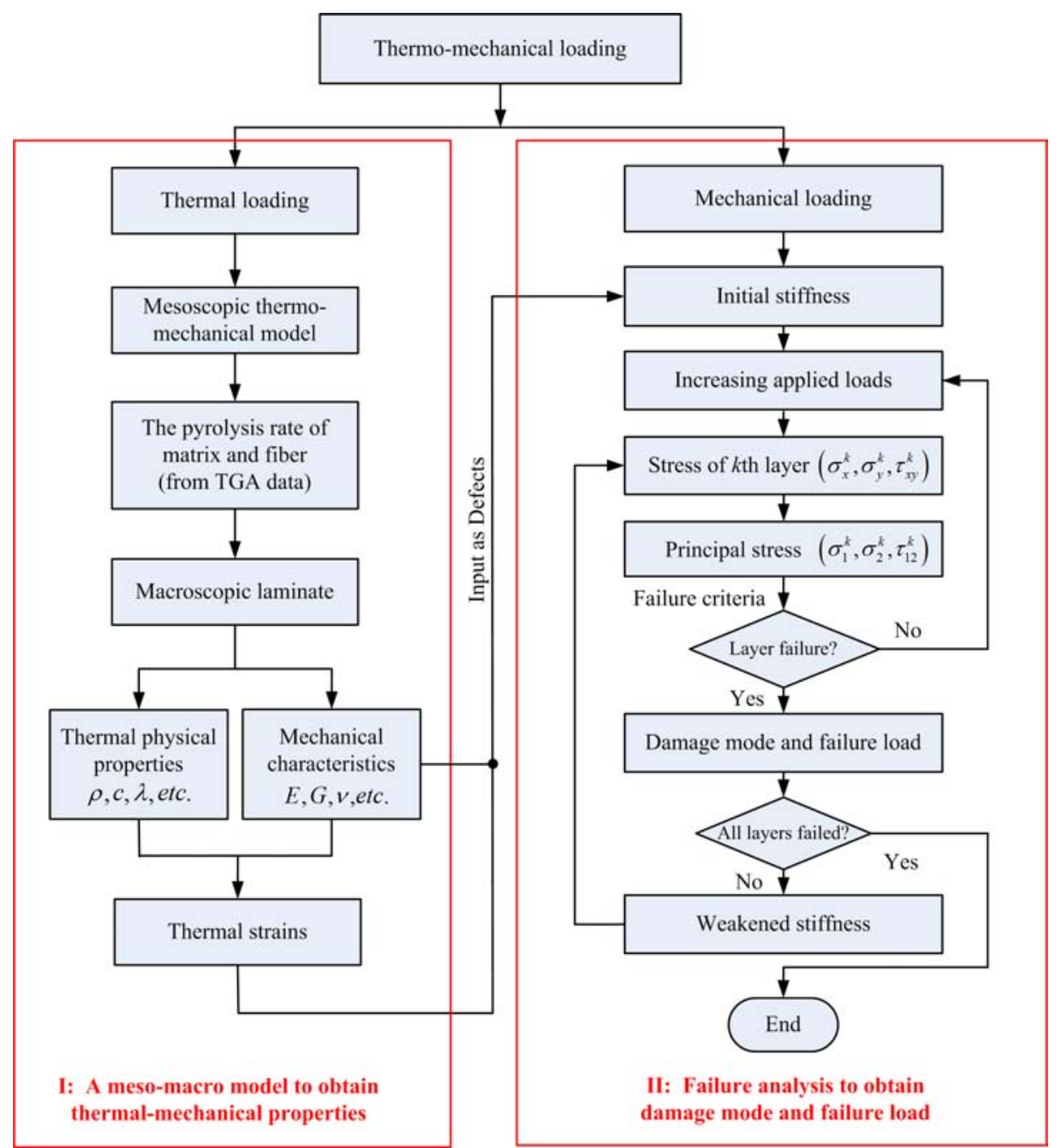

Fig. 2. Flowchart of failure analysis for laminated CFRP composites under thermo-mechanical loadings.

\subsection{General analytical approach}

Figure 2 gives the flowchart of failure analysis for laminated CFRP composites subjected to thermo-mechanical loadings. A two-step approach is adopted, i.e., the response of the composite under thermal loadings and the failure process under mechanical loadings.

In the first step, a mesoscopic to macroscopic model, considering thermal degradation of both matrix and fibers, is established to obtain the thermo-mechanical properties of the CFRP composite at high temperatures. The thermal pyrolysis rates of matrix and fiber are determined by TGA tests at nitrogen and oxidation 
environments. Based on the model, thermos-physical properties and mechanical properties are determined as functions of the temperature and the temperature rise rate.

In the second step, a progressive destruction program based on the classical laminate theory is built, and thermo-mechanical properties of the decomposed composite are input as defects to the initial stiffness matrix. For laminated composites, the prediction of failure depends on stress in each single layer. The progressive failure process and failure mode is predicted by the Tsai-Hill criterion.

\section{Thermo-Mechanical Model of CFRP Laminates}

\subsection{Meso-macro model}

For the CFRP laminate composed of continuous fiber and polymer matrix, a mesomacro model of multi-level structures is adopted to obtain the thermo-mechanical properties of the laminate including thermal decomposition effects, as depicted in Fig. 3. The model is basically followed that is proposed by Dimitrienko [1997].

When the unidirectional composite is heated to the pyrolysis temperature, its mass decreases due to intensive decomposition gas generated and escaped from the matrix and fiber. As a result, the thermally decomposed matrix involves phases of original polymer (subscript " $b$ "), pyrolytic residuum $(p)$ and pore $(g)$; whereas the fibers involve phases of crystalline $(l)$, amorphic $(a)$ and pore $(g)$ [Dimitrienko, 1997]. Assuming that the volume of composite material remains constant during the thermal decomposition, the pyrolysis process can be expressed by the volume concentrations of the phases. Thus, the physical and mechanical properties of unidirectional material can be expressed by the volume concentrations of the phases under high temperatures.

\subsection{Thermal physical properties}

Based on constant volume assumption, the density of decomposed composite material can be used to characterize mass loss, which is written as

$$
\begin{aligned}
\rho & =\bar{\rho}_{f}+\bar{\rho}_{m} \\
\bar{\rho}_{f} & =\rho_{f} \varphi_{f} \\
\bar{\rho}_{m} & =\rho_{b} \varphi_{b}+\rho_{p} \varphi_{p} \\
\varphi_{p} & =\frac{\rho_{b}}{\rho_{p}}\left(1-\varphi_{b}\right)(1-\Gamma) \\
\varphi_{a} & =\frac{\varphi_{f}-\varphi_{f}^{0}\left(1-\Gamma_{f}\right)}{\Gamma_{f}}, \quad \varphi_{a}+\varphi_{l}=\varphi_{f}
\end{aligned}
$$

where $\rho$ and $\varphi$ are the densities of the phases and the volume concentrations of the phases, respectively, $\bar{\rho}_{f}$ and $\bar{\rho}_{m}$ are the average density of the decomposed fiber and 


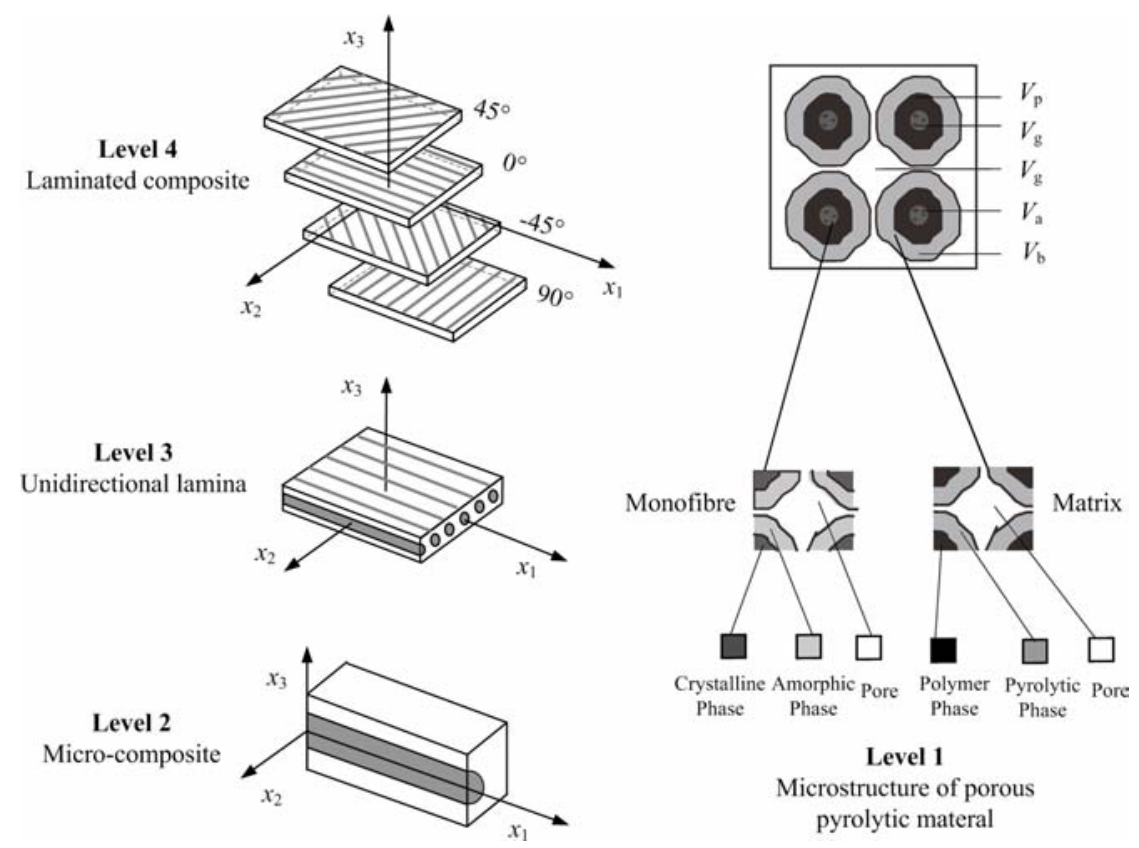

Fig. 3. Schematic diagram of a multi-level structure of laminated composite at high temperatures.

matrix. The superscript " 0 " means the original value, and the subscript is the abbreviation of each phase, where $f$-fibers (subscript " $f$ "), $b$-polymer phase (subscript " $b$ "), p-pyrolytic residuum phase (subscript " $p$ "), l-crystalline phase (subscript "l"), a-amorphic phase (subscript " $a$ "), g-pore phase (subscript " $g$ "). $\Gamma$ and $\Gamma_{f}$ are the gasification coefficients of the matrix and fibers determined by experiments.

The mass loss of composite material due to thermal decomposition can be expressed by the Arrhenius kinetic formula [Chen et al., 2010; Torre et al., 1998]

$$
\frac{\partial\left(m / m_{0}\right)}{\partial t}=-J_{0}\left(\frac{m-m_{r}}{m_{0}}\right)^{n} \exp \left(-\frac{E_{A}}{R \theta}\right)
$$

where $J_{0}, E_{A}$ and $n$ are the pre-exponential factor, activation energy of the thermal decomposition process, and order of the reaction. $m_{0}$ is the initial mass of the material, $m$ is the mass of the material at the thermal decomposition time, $m_{r}$ is the mass remained after thermal decomposition, $R$ is the gas constant, and $\theta$ is temperature.

Coupling with Eqs. (3.1)-(3.6), changing rates of volume concentrations of the polymer matrix and carbon fiber can be described with the Arrhenius forms

$$
\frac{\partial \varphi_{b}}{\partial t}=-J_{b} \varphi_{b}^{n} \exp \left(-\frac{E_{A b}}{R \theta}\right)
$$




$$
\begin{aligned}
& \frac{\partial \varphi_{f}}{\partial t}=-J_{f}\left[\varphi_{f}-\varphi_{f}^{0}\left(1-\Gamma_{f}\right)\right]^{n} \exp \left(-\frac{E_{A f}}{R \theta}\right) \\
& \varphi_{f}+\varphi_{b}+\varphi_{p}+\varphi_{g}=1
\end{aligned}
$$

where $J_{b}$ and $J_{t}$ are the pre-exponential factor for the polymer matrix and fibers respectively, and $E_{A b}$ and $E_{A f}$ are the activation energy of thermal decomposition for the polymer matrix and carbon fiber, respectively.

\subsection{Mechanical properties}

Under high temperatures, the elastic characteristics of unidirectional material depend essentially on temperature $\theta$ and heating time $t$. At elevated temperatures, both the matrix and fibers subject to thermal degradation. However, the thermostability of fibers is much better than that of polymer matrix, and the elastic modulus of fibers is also much higher. Therefore, for a single layer, the mechanical properties along fibers (or in the longitudinal direction) are determined by the pyrolysis parameters and elastic properties of fibers, whereas the properties of the direction perpendicular to fibers (or in the transverse direction) are determined by the polymer matrix properties and the matrix pyrolysis parameters. Elastic constants of lamina subject to thermal decomposition are

$$
\begin{cases}E_{1}=E_{f} a_{1} ; & E_{2}=E_{b} a_{2} \\ G_{12} \approx \frac{E_{2}}{2\left(1+\nu_{21}\right)}, & \nu_{21}=\mathrm{const}\end{cases}
$$

Function $a_{1}$ describes the changing elastic properties of the lamina in the longitudinal direction under heating, and $a_{2}$ describes those in the transverse direction. Expressions for $a_{1}, a_{2}$ are written in the form [Dimitrienko, 1997]

$$
\begin{aligned}
a_{1} & =a_{\theta 1}\left(s_{l}-s_{a}\right)+\left(1-s_{l}\right) n_{l}, \quad s_{l}=\left(1-\frac{\varphi_{f}-\varphi_{a}}{\varphi_{f}^{0}}\right)^{1 / 3} \\
s_{a} & =\left(1-\frac{\varphi_{f}}{\varphi_{f}^{0}}\right)^{1 / 3}, \quad n_{l}=\frac{E_{l}}{E_{a}} \\
a_{\theta 1} & =\exp \left(-b_{1} \Delta \bar{\theta}\right), \quad \Delta \bar{\theta}=\frac{\theta}{\theta_{0}}-1+\xi \int_{0}^{t} \exp [-\eta(t-\tau)]\left(\frac{\theta(\tau)}{\theta_{0}}-1\right) d \tau \\
a_{2} & =\left(\frac{s_{b}}{a_{\theta 2}\left(s_{p}^{2}-s_{b}^{2}\right)+m_{p}\left(1-s_{p}^{2}\right)}+\frac{s_{p}-s_{b}}{a_{\theta 2} s_{p}^{2}+m_{p}\left(1-s_{p}^{2}\right)}+\frac{1-s_{p}}{m_{p}}\right)^{-1}\left(1-\delta_{f}\right) \\
a_{\theta 2} & =\exp \left(-b_{2} \Delta \bar{\theta}\right), \quad m_{p}=\frac{E_{p}}{E_{b}}, \quad s_{b}=\left(\frac{\varphi_{g}}{\varphi_{b}^{0}}\right)^{1 / 3}, \\
s_{p} & =\left(\frac{\varphi_{b}^{0}-\varphi_{p}}{\varphi_{b}^{0}}\right)^{1 / 3}, \quad \delta_{f}=2\left(\frac{\varphi_{f}}{\pi}\right)^{1 / 2}
\end{aligned}
$$


where $s_{b}, s_{p}, s_{a}$ and $s_{l}$ represent the dimension of each phases after mapping, $\xi, \eta$, $b_{1}$ and $b_{2}$ are constants. Functions $a_{\theta 1}, a_{\theta 2}$ describe the changes of elastic features of the phases. Therefore, $a_{1}, a_{2}$ describe the contribution of each component of the composite to the mechanical properties during the thermal decomposition process.

\section{Failure Analysis}

\subsection{Effect of thermal decomposition}

In the Sec. 3, thermal physical and mechanical properties of the single layer composite materials based on the meso-macro model are obtained at high temperatures. These properties can be taken as macroscopic defects and substituted into the constitutive equations. Coupling with mechanical analysis program, damage modes and failure loadings of CFRP laminates can be achieved under different temperatures and heating rates.

\subsection{Stress state of single layer and failure criterion}

The single layer (or lamina) is the basic unit of the laminated composite. Compared with the other plane directions (1, 2 directions), the thickness ( 3 direction) of a single layer is very small, and can be taken as the plane stress state, i.e., $\sigma_{3}=0$, $\tau_{23}=\tau_{31}=0$. For orthotropic material, the stress-strain relationship for plane stress condition expressed in engineering constants of $Q_{i j}$ is

$$
\left\{\begin{array}{c}
\sigma_{1} \\
\sigma_{2} \\
\tau_{12}
\end{array}\right\}=\left[\begin{array}{ccc}
Q_{11} & Q_{12} & 0 \\
Q_{12} & Q_{22} & 0 \\
0 & 0 & Q_{66}
\end{array}\right]\left\{\begin{array}{l}
\varepsilon_{1} \\
\varepsilon_{2} \\
\gamma_{12}
\end{array}\right\}
$$

where

$$
\left.\begin{array}{l}
Q_{11}=\frac{E_{1}}{1-\nu_{12} \nu_{21}}, \quad Q_{22}=\frac{E_{2}}{1-\nu_{12} \nu_{21}} \\
Q_{12}=\frac{\nu_{21} E_{2}}{1-\nu_{12} \nu_{21}}=\frac{\nu_{12} E_{1}}{1-\nu_{12} \nu_{21}}, \quad Q_{66}=G_{12}
\end{array}\right\}
$$

Thus, the plane stress problem of orthogonal anisotropic single layer has four independent elastic constants $E_{1}, E_{2}, \nu_{12}, G_{12}$.

The Tsai-Hill criterion is adopted to determine whether the lamina is failed

$$
\frac{\sigma_{1}^{2}}{X^{2}}-\frac{\sigma_{1} \sigma_{2}}{X^{2}}+\frac{\sigma_{2}^{2}}{Y^{2}}+\frac{\tau_{12}^{2}}{S^{2}}=1
$$

where $X, Y$ and $S$ are longitudinal strength, transverse strength and shear strength respectively. The criterion fits for the composite whose tensile strength and compressive strength are the same. 


\subsection{Progressive damage analysis and equivalent elastic properties}

In the classical laminate theory, the subscript 1-2 is usually defined as main direction coordinate, and the subscript $x-y$ is global coordinate. So the stress-strain relationship of arbitrary direction by coordinate transformation can be obtained.

For symmetric laminated composites, geometric properties and material properties of each layer are symmetrical to the middle layer. Supposing the deformation of middle layer is $\varepsilon^{0}, \varepsilon^{0}, \gamma^{0}$, one gets the stress tensor of $k$ th layer at the elevated temperature

$$
\left\{\begin{array}{c}
\sigma_{x} \\
\sigma_{y} \\
\tau_{x y}
\end{array}\right\}_{k}=\left[\begin{array}{lll}
\overline{Q_{11}} & \overline{Q_{12}} & \overline{Q_{16}} \\
\overline{Q_{12}} & \overline{Q_{22}} & \overline{Q_{26}} \\
\overline{Q_{16}} & \overline{Q_{26}} & \overline{Q_{66}}
\end{array}\right]_{k}\left\{\begin{array}{c}
\varepsilon_{x}^{0}-\varepsilon_{x}^{\theta} \\
\varepsilon_{y}^{0}-\varepsilon_{y}^{\theta} \\
\gamma_{x y}^{0}-\gamma_{x y}^{\theta}
\end{array}\right\}_{k}
$$

where $\varepsilon^{\theta}, \varepsilon^{\theta}, \gamma^{\theta}$ are thermal strains due to the elevated temperatures.

It is proved that $\left[45^{\circ} / 0^{\circ} /-45^{\circ} / 90^{\circ}\right]_{2 S}$ laminate has quasi-isotropic property, therefore, the theoretically predicted equivalent Young's modulus and Poisson's ratio of the laminated composite can be derived

$$
\begin{aligned}
& E=\frac{\left(E_{1}+E_{2}\right)^{2}-4 \nu_{21}^{2} E_{2}^{2}+4 G_{12}\left(1-\nu_{12} \nu_{21}\right)\left(E_{1}+E_{2}+2 \nu_{12} \nu_{21}\right)}{\left[3 E_{1}+3 E_{2}+2 \nu_{21} E_{2}+4 G_{12}\left(1-\nu_{12} \nu_{21}\right)\right]\left(1-\nu_{12} \nu_{21}\right)} \\
& \nu=\frac{E_{1}+E_{2}+6 \nu_{21} E_{2}-4 G_{12}\left(1-\nu_{12} \nu_{21}\right)}{3 E_{1}+3 E_{2}+2 \nu_{21} E_{2}+4 G_{12}\left(1-\nu_{12} \nu_{21}\right)}
\end{aligned}
$$

A failure analysis code based on MATLAB platform is developed to obtain the damage mode and failure load of each lamina at high temperatures, which is following the procedure depicted in Fig. 2 and described in Sec. 2. The detail is as following: First, calculate stiffness and flexibility of laminates and each single layer based on the decomposed features and ply orientation, and express the stress of each single layer at a given applied load. Second, compare the stress status in each layer with the Tsai-Hill criterion, to determine if any layer is failed. If no layer is failed, the applied load is increased. Otherwise, the first-ply failure can be obtained. Thirdly, the stress status of first failure layer can be calculated. Compared with the strength in different orientation, the weakened stiffness of the first failure layer based on the failure modes can be obtained. For example, comparing the stress status of the failure layer with the strength, the result finds that the normal stress in the transverse direction is larger than the strength of that direction. Then mechanical properties in the transverse direction are weakened to zero, and mechanical properties in other directions still maintain. Fourth, recalculate laminates stiffness and check whether there is a chain of layer damage with the weakened stiffness. If there is no further damage, increase applied loadings and repeat the previous calculation progress. Increase the applied load till the last layer failure is happened. Therefore, the whole damage analysis of laminates is obtained. 


\section{Results and Discussions}

\subsection{Thermal decomposition parameters}

The thermal decomposition kinetic parameters in Eqs. (3.7) and (3.8) can be determined from TGA tests. Figures 4 and 5 are the TGA results of two laminated composites in nitrogen environment and oxidation environment, respectively. Thermal decomposition behaviors of CCF300/BA9916 laminates and T700/BA9916 laminates in a certain condition are basically the same. The difference of TGA for the two laminates is mainly due to different component percentage, for CCF300/BA9916, the initial volume fraction is $\varphi_{f}^{0}=0.420, \varphi_{b}^{0}=0.570, \varphi_{g}^{0}=0.010$, whereas for $\mathrm{T} 700 / \mathrm{BA} 9916, \varphi_{f}^{0}=0.533, \varphi_{b}^{0}=0.476, \varphi_{g}^{0}=0$. For the same laminate, the lower the heating rate, the more complete the laminate is decomposed, or the lower temperature the laminate is completely decomposed. In the Nitrogen environment, the fiber is hardly decomposed, therefore at the end of TGA test more than half of the

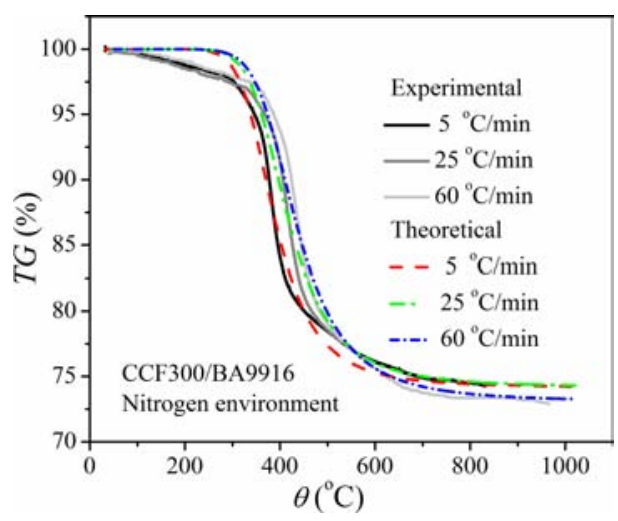

(a) CCF300/BA9916 $\left[45^{\circ} / 0^{\circ} /-45^{\circ} / 90^{\circ}\right]_{2 S}$ laminate

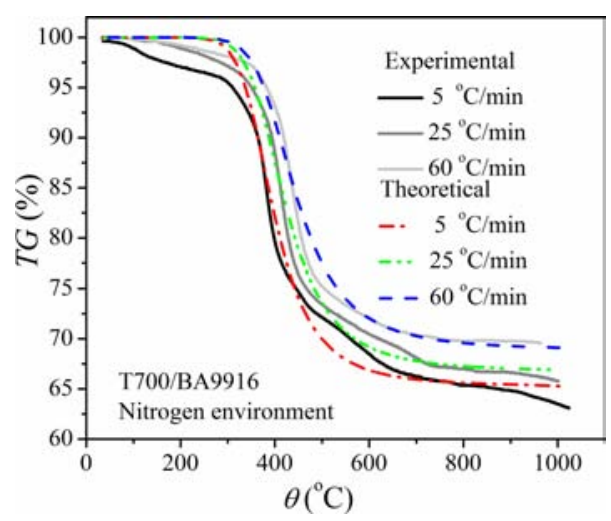

(b) T700/BA9916 $\left[45^{\circ} / 0^{\circ} /-45^{\circ} / 90^{\circ}\right]_{2 S}$ laminate

Fig. 4. TGA of CFRP laminates at different heating rates, in the nitrogen environment. 


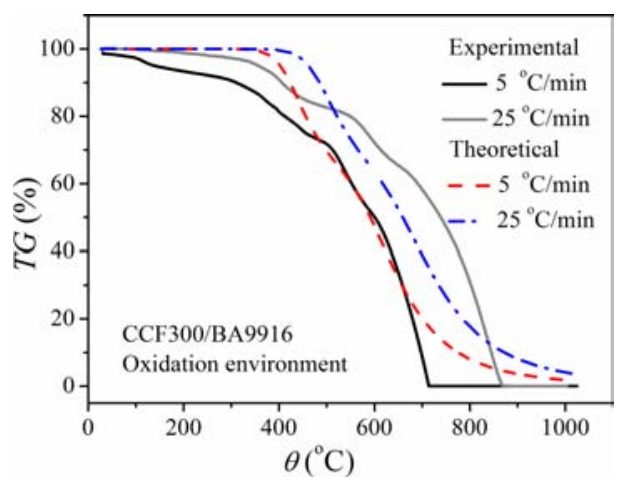

(a) CCF300 / BA9916 $\left[45^{\circ} / 0^{\circ} /-45^{\circ} / 90_{2 \mathrm{~S}}^{\circ}\right.$ laminate

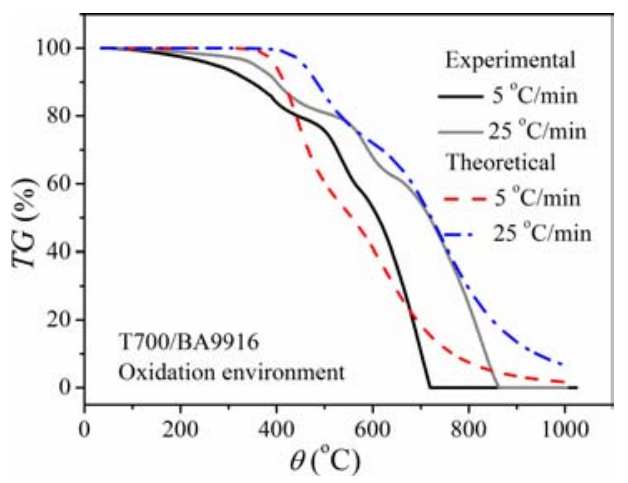

(b) T700/BA9916 $\left[45^{\circ} / 0^{\circ} /-45^{\circ} / 90^{\circ}\right]_{2 \mathrm{~S}}$ laminate

Fig. 5. TGA of CFRP laminates at different heating rates, in the oxidation environment.

original mass remains. The decomposition behavior of the composite in an oxidation environment is more complex, due to the oxidizing process of carbon fibers. At the end of TGA test, both the matrix and fibers are completely decomposed with zero mass left.

Based on the theory of pyrolysis, thermal decomposition kinetic parameters are determined by TGA. According to TGA data, a multi-level decomposition model is used to calculate the thermal decomposition kinetic parameters for various CFRP laminates under different environments. For CCF300/BA9916 and T700/BA9916, material constants describing the degradation of density and heat capacity can be obtained from Dimitrienko [1997]. According to references, the carbon fiber is hardly decomposed until the temperature reaches $3000^{\circ} \mathrm{C}$ in the nitrogen environment. Thus, the mass loss of the matrix nearly equals to the mass loss of the composite. The order of the reaction is assumed to be equal to 3 . Therefore, the thermal decomposition kinetic parameters of the matrix can be obtained from TG tests in the nitrogen environment. The difference of fitted TG curve of the matrix and TG test of CFRP is attributed to the decomposition of the fiber. Although it is a minor 
Table 1. Thermal decomposition kinetic parameters.

\begin{tabular}{lccc}
\hline Environment & constants & CCF300/BA9916 & T700/BA9916 \\
\hline Nitrogen environment & $n$ & 3 & 3 \\
& $E_{A b}(\mathrm{~J} / \mathrm{kg})$ & $1.02 \times 10^{5}$ & $1.05 \times 10^{5}$ \\
& $J_{b}\left(\mathrm{~kg} \cdot \mathrm{m}^{-3} \cdot \mathrm{s}^{-1}\right)$ & $4.30 \times 10^{6}$ & $4.30 \times 10^{6}$ \\
& $\Gamma$ & 0.7 & 0.7 \\
& $E_{A f}(\mathrm{~J} / \mathrm{kg})$ & $2.0 \times 10^{5}$ & $2.0 \times 10^{5}$ \\
& $J_{f}\left(\mathrm{~kg} \cdot \mathrm{m}^{-3} \cdot \mathrm{s}^{-1}\right)$ & $3.0 \times 10^{6}$ & $3.0 \times 10^{6}$ \\
& $\Gamma_{f}$ & 0.05 & 0.05 \\
Oxidation environment & $n$ & 3 & 3 \\
& $E_{A b}(\mathrm{~J} / \mathrm{kg})$ & $1.73 \times 10^{5}$ & $1.75 \times 10^{5}$ \\
& $J_{b}\left(\mathrm{~kg} \cdot \mathrm{m}^{-3} \cdot \mathrm{s}^{-1}\right)$ & $6.0 \times 10^{6}$ & $6.0 \times 10^{6}$ \\
& $\Gamma$ & 1 & 1 \\
& $E_{A f}(\mathrm{~J} / \mathrm{kg})$ & $1.5 \times 10^{5}$ & $1.55 \times 10^{5}$ \\
& $J_{f}\left(\mathrm{~kg} \cdot \mathrm{m}^{-3} \cdot \mathrm{s}^{-1}\right)$ & $3.2 \times 10^{6}$ & $4.2 \times 10^{6}$ \\
& $\Gamma_{f}$ & 1 & 1 \\
\hline
\end{tabular}

portion of decomposition, it should be considered. The order of magnitude about the fiber decomposition kinetic parameters can be obtained from references. Based on the decomposition equations, the thermal decomposition kinetic parameters of the fiber can be obtained in the nitrogen environment. Based on the results in the nitrogen environment and assuming the gasification coefficients of the matrix and fibers both equal to 1 , the thermal decomposition kinetic parameters for both epoxy matrix and carbon fiber in the oxidation environment can be obtained. The pre-exponential factor and the activation energy of thermal decomposition for both epoxy matrix and carbon fiber in Eqs. (3.7) and (3.8) can be obtained from TGA. The parameters are listed in Table 1.

Figures 4 and 5 show good agreements between experimental and theoretical TG results, which validate the reliability of thermal decomposition model.

Depending on the thermal decomposition kinetic parameters, the weight percentage of each component (fiber, polymer and residual char) can be obtained during the whole thermal decomposition process. Taking CCF300/BA9916 at heating rate of $5^{\circ} \mathrm{C} / \mathrm{min}$ in a nitrogen environment as an example, the results are shown in Fig. 6. It can be seen that the fiber mass remains nearly constant, which means thermal decomposition of carbon fibers can hardly occur in a nitrogen environment. The thermal decomposition behavior is mainly determined by the pyrolysis of epoxy matrix, whose pyrolysis temperature is about $230^{\circ} \mathrm{C}$. In addition, the heating rate significantly affects the thermal properties, since heating rate governs the rate and extent of thermal decomposition. At the lower heating rate, the thermal decomposition of composites is more complete when reaching the same temperature.

\subsection{Mechanical properties}

Elastic constants for the fiber and matrix are listed in Table 2. According to Eq. (3.10), theoretically predicted mechanical properties of T700/BA9916 lamina 


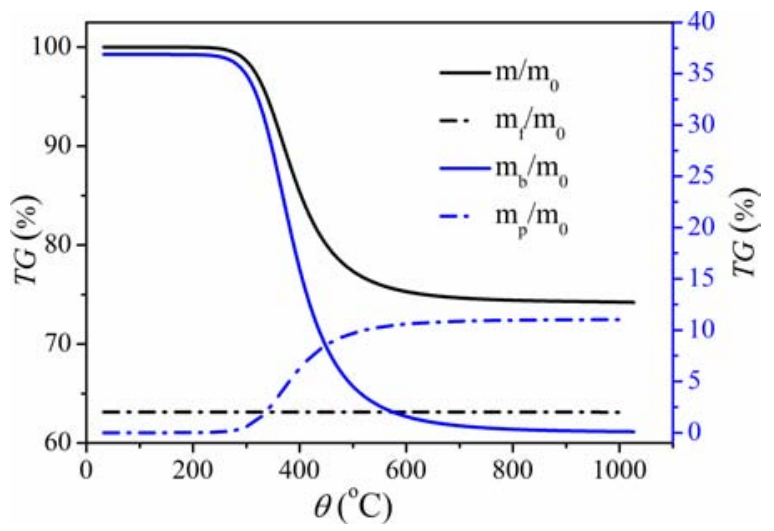

Fig. 6. Component percentage during the thermal decomposition process, TGA of CCF300/BA9916 at heating rate of $5^{\circ} \mathrm{C} / \mathrm{min}$ in nitrogen environment.

Table 2. Material constants describing the mechanical properties of single layer.

\begin{tabular}{lcc}
\hline Constants & CCF300/BA9916 & T700/BA9916 \\
\hline$E_{b}(\mathrm{GPa})$ & 9.82 & 9.82 \\
$G_{b}(\mathrm{GPa})$ & 3.78 & 3.78 \\
$E_{f}(\mathrm{GPa})$ & 125.5 & 116.0 \\
$\nu_{21}$ & 0.30 & 0.30 \\
\hline
\end{tabular}

at elevated and high temperature can be obtained. Figure 7 illustrates the effects of heating rate on the elastic modulus of lamina. At low heating rate, i.e., $5^{\circ} \mathrm{C} / \mathrm{min}$, the lamina lost the stiffness at about $200^{\circ} \mathrm{C}$. Whereas, at high heating rate, i.e., $60^{\circ} \mathrm{C} / \mathrm{min}$, the lamina still possesses a certain of stiffness at about $400^{\circ} \mathrm{C}$. The degradation of longitudinal elastic modulus $E_{1}$, which is mainly affected by the thermal stability of carbon fibers, is less obvious than that of transverse and shear modulus, which are dominated by epoxy matrix pyrolysis. Again, the heating rate significantly affects the mechanical properties, due to the different thermal decomposition rate and extent.

\subsection{Thermal deformation}

Under high temperatures, thermal deformation of composite lamina is nonlinear upon temperature $\theta$, due to the pyrolysis of the polymer matrix accompanied by chemical contraction [Dimitrienko, 1999].

$$
\varepsilon_{T i}=\alpha_{i} \frac{\varphi_{m}}{\varphi_{m}^{0}}\left(\theta-\theta_{0}\right)+\alpha_{c i} \int_{0}^{t} \frac{\varphi_{p}}{\varphi_{p}^{*}} \theta(\tau) d \tau-\alpha_{i}^{*} \frac{\varphi_{p}}{\varphi_{p}^{*}}
$$

where $\varepsilon_{T i}$ is the thermal strain of the composite in the $i$ direction at room temperature, $\alpha_{i}$ is thermal expansion coefficient, $\alpha_{c i}$ is thermal expansion coefficient of the composite at high temperatures, $\alpha_{i}^{*}$ is chemical contraction coefficient during 


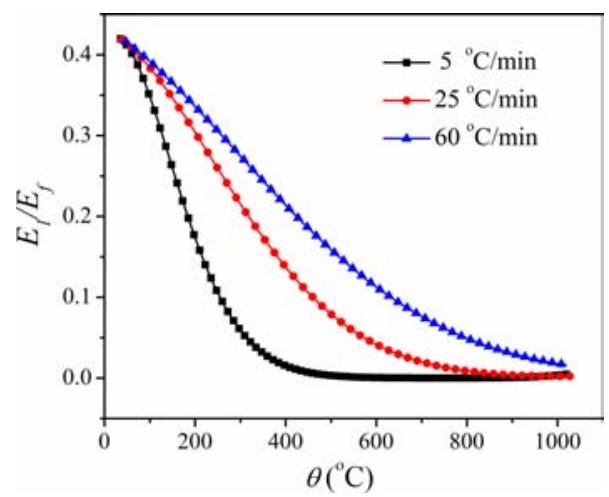

(a) Longitudinal elastic modulus

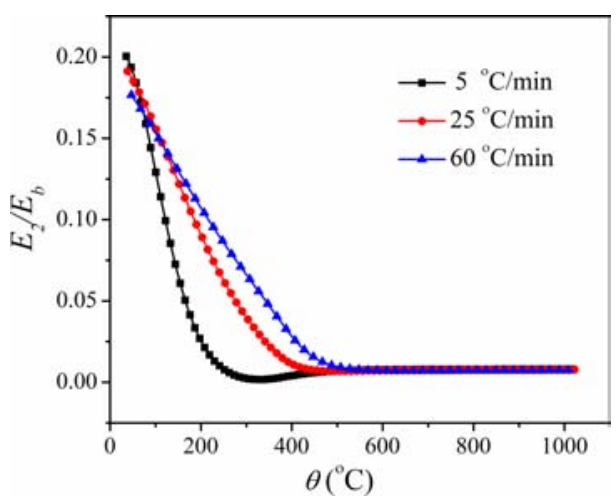

(b) Transverse elastic modulus

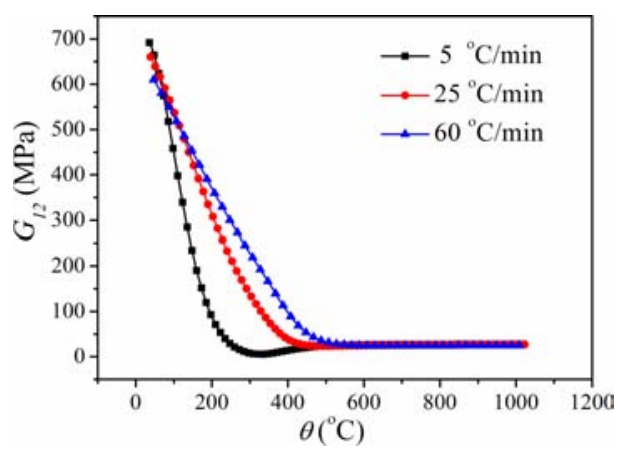

(c) Shear modulus

Fig. 7. Variation of elastic constants of single layer with thermal decomposition process, T700/BA9916. 
Table 3. Material constants describing thermal deformation of single layer composites.

\begin{tabular}{lcc}
\hline Constants & Longitudinal direction $(i=1)$ & Transverse direction $(i=2)$ \\
\hline$\alpha_{i}\left(10^{-6} \mathrm{~K}^{-1}\right)$ & 0.2 & 20 \\
$\alpha_{c i}\left(10^{-6} \mathrm{~K}^{-1}\right)$ & 1.5 & 4.0 \\
$\alpha_{i} *\left(10^{-6} \mathrm{~K}^{-1}\right)$ & 0.01 & 0.16 \\
\hline
\end{tabular}

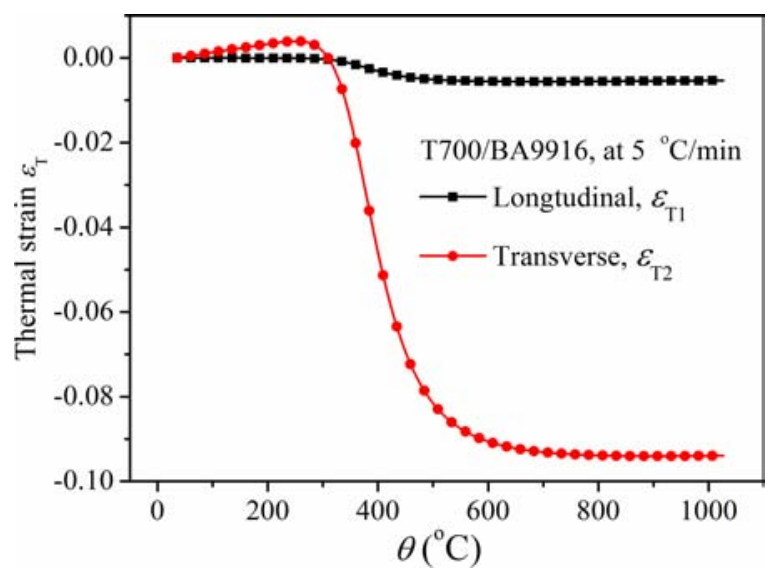

Fig. 8. Thermal strain of single layer during the thermal decomposition process, T700/BA9916.

the pyrolysis process. From Eq. (5.1), thermal deformation of composite is mainly composed of three parts at high temperatures. The first item is thermal expansion of the polymer matrix before pyrolysis, the second item represents the thermal deformation of new phases produced in the pyrolysis process, and the last item is chemical contraction due to breaking of chemical bonds.

Figure 8 gives the longitudinal and transverse thermal strains of T700/BA9916 lamina at the heating rate of $5^{\circ} \mathrm{C} / \mathrm{min}$. Material constants used to describe the thermal deformation are listed in Table 3. A slight increase in thermal strains is observed when the temperature is below $300^{\circ} \mathrm{C}$, due to thermal expansion of polymer matrix before pyrolysis. After that, the transverse strain drops significantly, due to the chemical shrinkage of polymer matrix in the pyrolysis process, whereas the drop in longitudinal strain is not obvious. Figure 9 is the comparison of thermal deformation between two CFRP composites at different heating rate. No significant difference is found between these two composites, whereas the heating rate may obviously influence the thermal shrinkage during the pyrolysis process.

\subsection{Progressive failure}

According to the flowchart given in Fig. 2, the failure process of each lamina and the failure mode of the laminated CFRP can be obtained. Figure 10 presents failure modes of CCF300/BA9916 $\left[45^{\circ} / 0^{\circ} /-45^{\circ} / 90^{\circ}\right]_{2 \mathrm{~S}}$ laminate at different temperatures 


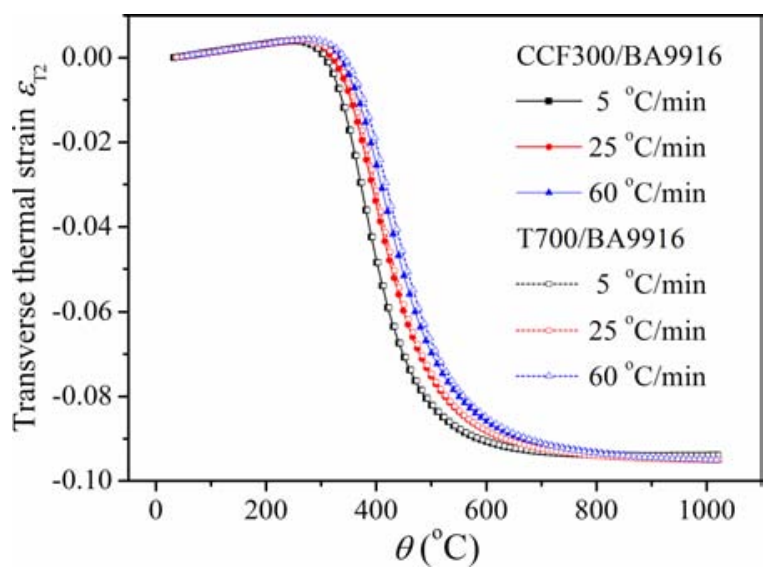

Fig. 9. Comparison of thermal strains of two CFRP composites during thermal decomposition process.

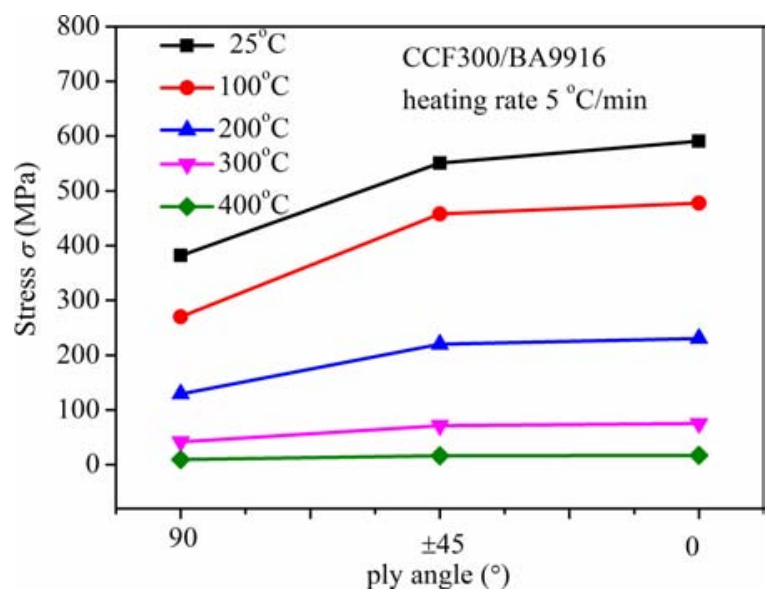

Fig. 10. Progressive Failure of CCF300/BA9916 $\left[45^{\circ} / 0^{\circ} /-45^{\circ} / 90^{\circ}\right] 2 S$ laminate at heating rate $5^{\circ} \mathrm{C} / \mathrm{min}$ under tensile loading in a nitrogen environment.

under tensile loading, which is heated at a rate of $5^{\circ} \mathrm{C} / \mathrm{min}$ in a nitrogen environment. Failure modes at high temperatures as while as at room temperature are similar, the laminate fails in the sequence of ply angle of $90^{\circ} \rightarrow \pm 45^{\circ} \rightarrow 0^{\circ}$, which typically means the progressive fracture mechanisms of matrix cracking, delamination and fiber breaking. Figure 11 gives failure strength of CCF300/BA9916 and T700/BA9916 laminates at the heating rate of $5^{\circ} \mathrm{C} / \mathrm{min}$. It is found that as the temperature increases, the failure strength decreases nonlinearly. Although at room temperature the failure strength of T700/BA9916 is significantly higher than that of CCF300/BA9916, it drops more obviously as temperature rises. This may due to the slow heating rate, at which both matrix and fibers are nearly decomposed when 


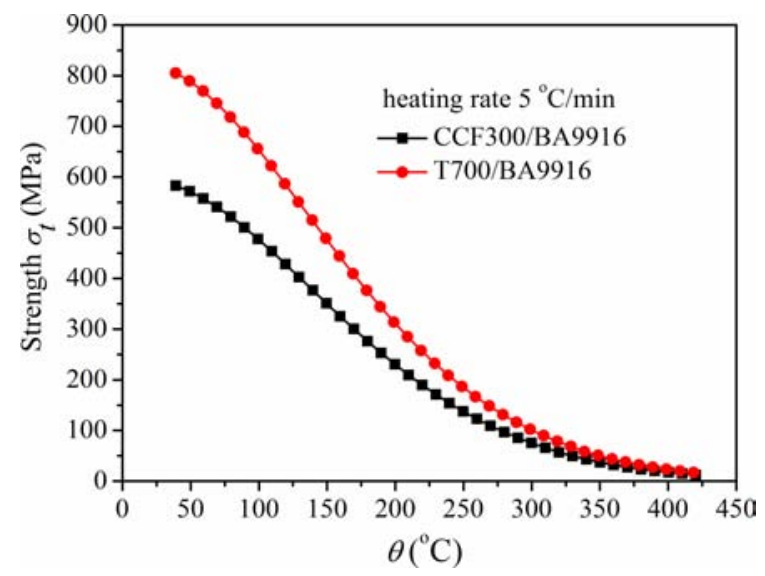

Fig. 11. Failure strength of two CFRP composites at heating rate $5^{\circ} \mathrm{C} / \mathrm{min}$ under tensile loading.

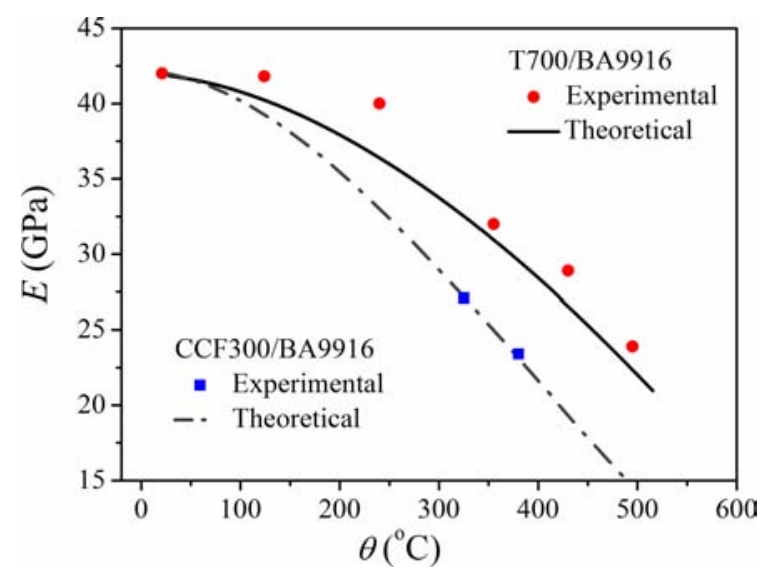

Fig. 12. Comparison of Young's modulus between theretical and experimental results, heating rate of about $100^{\circ} \mathrm{C} / \mathrm{min}$.

the temperature is high. At the temperature above $400^{\circ} \mathrm{C}$, both of the two CFRP composites lose the capability of load-bearing.

\subsection{Comparison with experiments}

Figures 12 and 13 present the comparison of experimental results and analytical results on the Young's modulus and failure strength. The experiments are carried out at the heating rate of about $100^{\circ} \mathrm{C} / \mathrm{min}$ in an oxidation environment. In the analytical model, the parameters in Eq. (3.11) for T700/BA9916 laminate are listed in Table 4.

In comparison, the analytical predicted Young's modulus is in good agreement with the experimental data, whereas the difference in the failure strength 


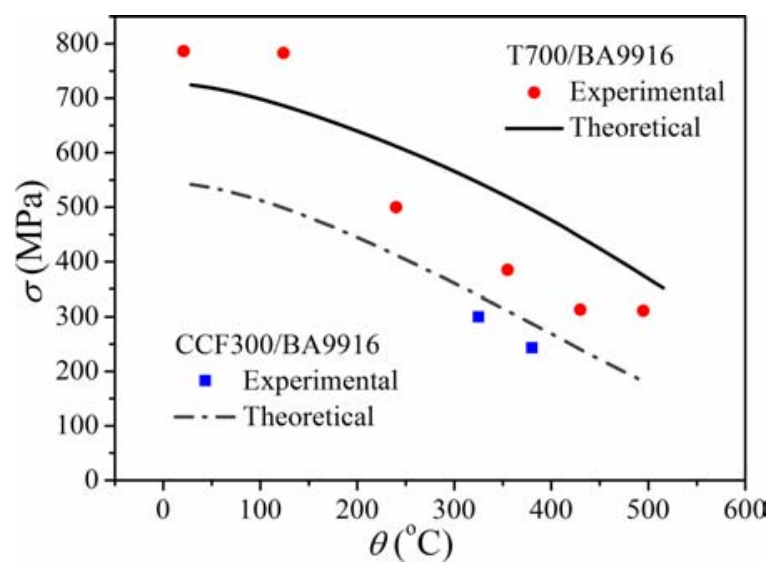

Fig. 13. Comparison of failure strength between theoretical and experimental results, heating rate of about $100^{\circ} \mathrm{C} / \mathrm{min}$.

Table 4. Constants of T700/BA9916 laminate describing mechanical properties.

\begin{tabular}{lcc}
\hline Environment & Constants & T700/BA9916 \\
\hline Oxidation environment & $\xi$ & $5.3 \times 10^{-2}$ \\
& $\eta$ & $2.5 \times 10^{-4}$ \\
& $b_{1}$ & 0.04 \\
& $b_{2}$ & 0.7 \\
\hline
\end{tabular}

of T700/BA9916 is a little big, which is about 20\%. Compared with Fig. 12, in which the two CFRP composites nearly lose strength at $300^{\circ} \mathrm{C}$, the T700/BA9916 still possesses a certain of strength and stiffness at the temperature of about $500^{\circ} \mathrm{C}$. This mainly dues to the different heat rate: the former is heated at a rate of $5^{\circ} \mathrm{C} / \mathrm{min}$, whereas the latter is heated at a rapid rate of $100^{\circ} \mathrm{C} / \mathrm{min}$. The T700/BA9916 composite is not completely thermal decomposed at such a high heating rate, although in an oxidation environment. From Figures 12 and 13 we can also find that upon heating, the Young's modulus and failure strength of T700/BA9916 composite are higher than those of CCF300/BA9916 at the same thermal environment. This mainly dues to the difference in the initial volume fraction of fiber, in CCF300/BA9916 $\varphi_{f}^{0}=0.420$, and in T700/BA9916 $\varphi_{f}^{0}=0.533$. Since polymer matrix is more easily and rapidly decomposed, the mechanical behavior of CCF300/BA9916, which has higher volume fraction of matrix, degrades more obviously at high heating rates.

\subsection{Comparison with other theories}

The statistical model proposed by Mahieux and Reifsnider [Mahieux and Reifsnider, 2001, 2002] assumes that increasing temperature has the effect of relaxing, increasing numbers of intermolecular bonds in the resin. The bond "strength" was assumed to follow the Weibull distribution as a function of temperature, giving rise to a simple 
functional relationship

$$
P(\theta)=P_{R}+\left(P_{U}-P_{R}\right) \exp \left(-\left(\frac{\theta}{\theta_{0}}\right)^{h}\right)
$$

where $P(\theta)$ is the mechanical property at temperature $\theta, P_{U}$ is the unrelaxed values of that property (i.e., before glass transition, generally at room temperature), and $P_{R}$ is the relaxed property (i.e., after the glass transition process, high temperature). $\theta_{0}$ is the relaxation temperature and $h$ is the Weibull exponent. Mahieux and Reifsnider have used this relationship successfully to fit modulus versus temperature data for several polymers. Bisby's model is provided as

$$
P(\theta)=P_{U}\left(\left(\frac{1-a}{2}\right) \tanh (-b(\theta-c))+\left(\frac{1+a}{2}\right)\right)
$$

where $a$ is an assumed constant, with the coefficients $b$ and cobtained by least-squares regression of test databases. In some way, $a$ is similar to the parameter $P_{R}$.

Gibson et al., in a real sense, proposed a theoretical model to predict the mechanical properties of laminated composite for elevated temperatures up to $200^{\circ} \mathrm{C}$. The model uses the output from a previously reported thermal ablative model in conjunction with laminate theory. The function considers the content of thermal damage as following form

$$
P(\theta)=\left(\frac{P_{U}+P_{R}}{2}-\frac{P_{U}-P_{R}}{2} \tanh \left(K\left(\theta-\theta_{g}\right)\right)\right) R_{m}^{r}
$$

where $K$ is a constant describing breadth of the relaxation process, and $\theta_{g}$ is the mechanically determined glass transition temperature. $R_{m}$ is the residual resin content (between 0 and 1 ) and $r$ is a constant that depends on the stress state (i.e., $r=0$ for tensile strength in FRPs).

Comparisons of these different models and present theory are plotted in Figs. 14 and $15 . T_{g}$ for the present $\mathrm{CFRP}$ is about $215^{\circ} \mathrm{C}$, thus values of parameters being used in each model are listed in Table 5.

From Figs. 14 and 15, it can be found that Bisby's model is similar to Gibson's model. All of the three models can successfully predict the reduction of tensile

Table 5. The parameter values of different models.

\begin{tabular}{lcc}
\hline Model & Property & Coefficients \\
\hline Mahieux and Reifsnider & Tensile strength & $P_{R}=0.498 P_{\mathrm{U}}, \theta_{0}=215^{\circ} \mathrm{C}, h=13$ \\
& Elastic modulus & $P_{R}=0.760 P_{\mathrm{U}}, \theta_{0}=215^{\circ} \mathrm{C}, h=11$ \\
Bisby et al. & Tensile strength & $a=0.498, b=0.095, c=215^{\circ} \mathrm{C}$ \\
& Elastic modulus & $a=0.760, b=0.0930, c=215^{\circ} \mathrm{C}$ \\
Gibson et al. & Tensile strength & $P_{R}=0.498 P_{U}, K=0.0120, \theta_{g}=215^{\circ} \mathrm{C}, r=0$ \\
& Elastic modulus & $P_{R}=0.760 P_{\mathrm{U}}, K=0.00836, \theta_{g}=215^{\circ} \mathrm{C}, r=0$ \\
\hline
\end{tabular}




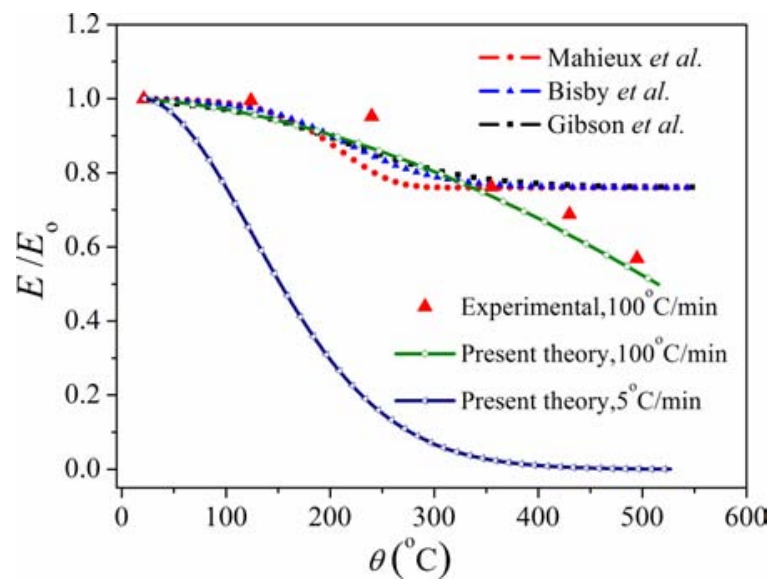

Fig. 14. Comparison of Young's modulus between previous model and present theory, in the oxidation environment (T700/BA9916).

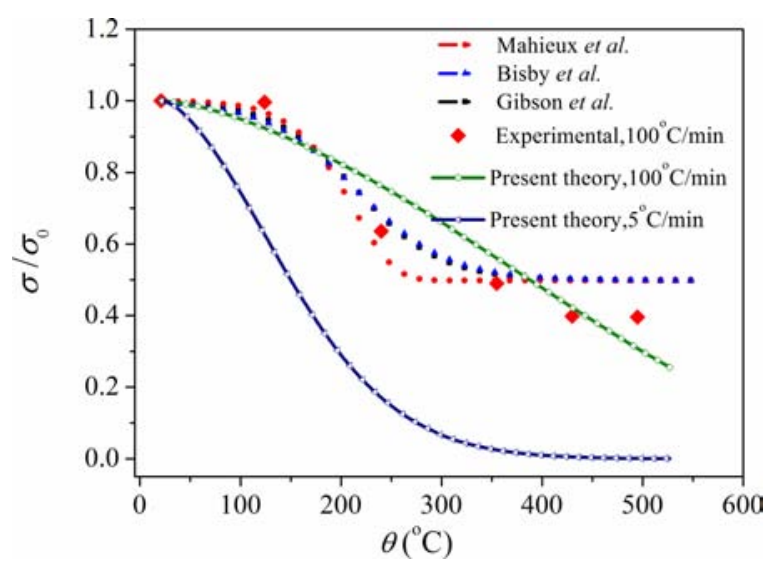

Fig. 15. Comparison of tensile strength between previous model and present theory, in the oxidation environment (T700/BA9916).

strength and elastic modulus below $200^{\circ} \mathrm{C}$. However, these models cannot accurately describe the mechanical properties of CFRP above $400^{\circ} \mathrm{C}$. In addition, it should be noted that previous models do not include the influence of heating rates. As plotted in Figs. 14 and 15, the mechanical behavior of CFRP predicted by present theory at heating rate of $5^{\circ} \mathrm{C} / \mathrm{min}$ is significantly different from that of $100^{\circ} \mathrm{C} / \mathrm{min}$ : the former share the similar morphology as those predicted by previous models, whereas almost lost mechanical capability at about $300^{\circ} \mathrm{C}$; the latter has no obvious relaxation stage due to the rapid heating rate, which is basically verified by the experimental results. Figures 16 and 17 illustrate the theoretical predicted degradation behavior of Young's modulus and tensile strength at different heating rate. It can be found that, in a moderate temperature, e.g., $300^{\circ} \mathrm{C}$, the residual mechanical properties 


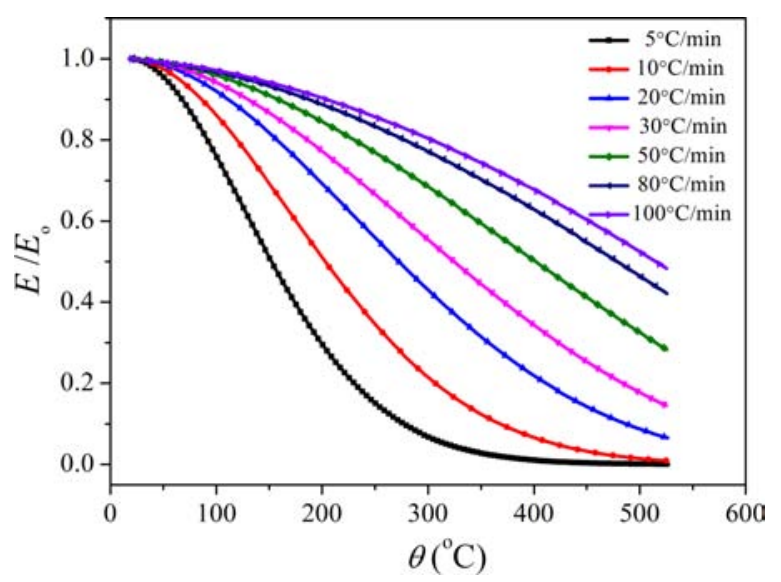

Fig. 16. Degradation of Young's modulus at different heating rate.

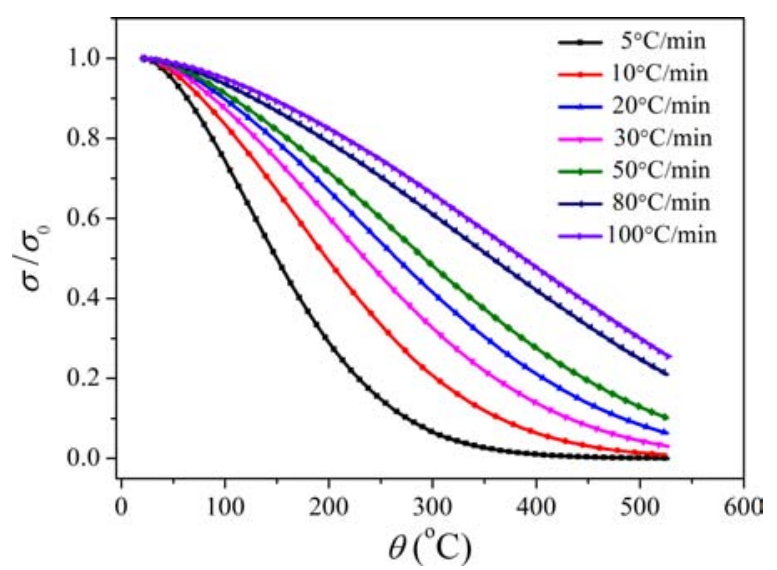

Fig. 17. Degradation of tensile strength at different heating rate.

are around $80 \%$ at the heating rate of $100^{\circ} \mathrm{C} / \mathrm{min}$, whereas they are below $10 \%$ at the heating rate of $5^{\circ} \mathrm{C} / \mathrm{min}$.

\section{Conclusion}

To model the failure behavior of laminated CFRP composite subjected to combining thermal and mechanical loading, a multi-level structure model is needed to bridge the gap between mesoscopic level, which includes physical and chemical process of thermal decomposition in fibers and matrix of single cell, and macroscopic level, which includes thermo-mechanical degradation in single layer and the entire laminate. Coupling the multi-level thermal-mechanical model with the progressive 
mechanical destruction program, the present theory can predict the failure behavior of laminated CFRP made of CCF300/BA9916 and T700/BA9916 at elevated and high temperature. The results show that the higher the heating rate, the less degraded are the thermo-mechanical properties and failure strength at a given temperature. Young's modulus and tensile failure strength of T700/BA9916 are higher than those of CCF300/BA9916 at high temperatures, due to the higher volume fraction of carbon fibers, which are less weakened in thermal environment.

\section{Acknowledgments}

Finical supports from National Natural Science Foundation of China (Grant Nos. 91016025, 11332011 and 11472276), and Project of Chinese Academy of Sciences, are gratefully acknowledged.

\section{References}

Bahramian, A. R., Kokabi, M., Famili, M. H. N. et al. [2006] "Ablation and thermal degradation behaviour of a composite based on resol type phenolic resin: Process modeling and experimental," Polymer 47, 3661-3673.

Bisby, L. A., Green, M. F. and Kodur, V. K. R. [2005] "Modeling the behavior of fiber reinforced polymer-confined concrete columns exposed to fire," Journal of Composites for Construction 9, 15-24.

Bai, Y., Vallee, T. and Keller, T. [2008] "Modeling of thermal responses for FRP composites under elevated and high temperatures," Composites Science and Technology 68, 4756 .

Bai, Y. and Keller, T. [2009] "Modeling of mechanical response of FRP composites in fire," Composites Part A: Applied Science and Manufacturing 40, 731-738.

Chen, M., Jiang, H. and Liu, Z. [2010] "Determination of thermal decomposition kinetic parameters of glass-fiber/epoxy composite," High Power Laser and Particle Beams 22, 1969-1972.

Dimitrienko, Y. [1997] "Modelling of the mechanical properties of composite materials at high temperatures.1. Matrix and fibers," Applied Composite Materials 4, 219-237.

Dimitrienko, Y. [1999] Thermomechanics of Composites under High Temperatures (Kluwer, Moscow).

Feih, S., Mathys, Z., Gibson, A. G. et al. [2007] "Modelling the tension and compression strengths of polymer laminates in fire," Composites Science and Technology 67, 551564 .

Gadade, A. M., Lal, A. and Singh, B. N. [2016a] "Finite element implementation of Puck's failure criterion for failure analysis of laminated plate subjected to biaxial loadings," Aerospace Science and Technology 55, 227-241.

Gadade, A. M., Lal, A. and Singh, B. N. [2016b] "Accurate stochastic initial and final failure of laminated plates subjected to hygro-thermo-mechanical loadings using Puck's failure criteria," International Journal of Mechanical Sciences 114, 177-206.

Gibson, A. G., Wu, Y. S., Evans, J. T. et al. [2006] "Laminate theory analysis of composites under load in fire," Journal of Composite Materials 40, 639-658.

Gotsis, P. K., Chamis, C. C. and Minnetyan, L. [1998] "Prediction of composite laminate fracture: Micromechanics and progressive fracture," Composites Science and Technology 58, 1137-1149. 
Huang, Z. M. [2004] "A bridging model prediction of the ultimate strength of composite laminates subjected to biaxial loads," Composites Science and Technology 64, 395448.

Kandare, E., Griffin, G. J., Feih, S. et al. [2012] "Fire structural modelling of fibre-polymer laminates protected with an intumescent coating," Composites Part A: Applied Science and Manufacturing 43, 793-802.

Li, R., Kelly, D. and Crosky, A. [2002] "An evaluation of failure criteria for matrix induced failure in composite materials," Composite Structures 57, 385-391.

Mouritz, A. P., Feih, S., Kandare, E., Mathys, Z., Gibson, A. G., Des Jardin, P. E. et al. [2009] "Review of fire structural modelling of polymer composites," Composites Part A: Applied Science and Manufacturing 40, 1800-1814.

Mahieux, C. A. and Reifsnider, K. L. [2001] "Property modeling across transition temperatures in polymers: A robust stiffness-temperature model," Polymer 42, 3281-3291.

Mahieux, C. A. and Reifsnider, K. L. [2002] "Property modeling across transition temperatures in polymers: Application to thermoplastic systems," Journal of Materials Science 37, 911-920.

Rupnowski, P., Gentz, M. and Kumosa, M. [2006] "Mechanical response of a unidirectional graphite fiber/polyimide composite as a function of temperature," Composites Science and Technology 66, 1045-1055.

Soden, P. D., Hinton, M. J. and Kaddour, A. S. [1998] "Lamina properties, lay-up configurations and loading conditions for a range of fibre-reinforced composite laminates," Composites Science and Technology 58, 1011-1022.

Torre, L., Kenny, J. M. and Maffezzoli, A. M. [1998] "Degradation behaviour of a composite material for thermal protection systems part I - Experimental characterization," Journal of Materials Science 33, 3137-3143.

Wang, K., Young, B. and Smith, S. T. [2011] "Mechanical properties of pultruded carbon fibre-reinforced polymer (CFRP) plates at elevated temperatures," Engineering Structures 33, 2154-2161.

Zenkour, A. M., Allam, M. N. M. and Radwan, A. F. [2013] "Bending of cross-ply laminated plates resting on elastic foundations under thermo-mechanical loading," International Journal of Mechanics and Materials in Design 9, 239-251. 